
И ИНГИБИТОРА СИНТЕЗА ПРОСТАГЛАНДИНОВ ПРИ НЕДЕРЖАНИИ МОЧИ У ЖЕНЩИН

\author{
(C) Н.А. Осипова ${ }^{1}$, Д.А. Ниаури ${ }^{1}$, А.М. Гзгзянн ${ }^{1,2}$ \\ ${ }^{1}$ ФГБОУ ВПО «Санкт-Петербургский государственный университет», медицинский факультет, \\ Санкт-Петербург, Россия; \\ ${ }^{2}$ ФГБНУ «Научно-исследовательский институт акушерства, гинекологии и репродуктологии им. Д.О. Отта», \\ Санкт-Петербург, Россия
}

Для цитирования: Осипова Н.А., Ниаури Д.А., Гзгзян А.М. Клиническая эффективность применения антидиуретического гормона и ингибитора синтеза простагландинов при недержании мочи у женщин // Журнал акушерства и женских болезней. - 2018. - Т. 67. - № 2. - C. 40-51. doi: 10.17816/JOWD67240-51

Поступила в редакцию: 01.03.2018

Принята к печати: 03.04.2018

\begin{abstract}
- Aкmуальность. Вопросы патогенеза и способы лечения недержания мочи у женщин активно обсуждаются гинекологами, урологами и невропатологами. Недержание мочи чаще всего имеет мультифакторное происхождение: причины возникновения инконтиненции связывают, как правило, с нарушением функциональных механизмов удержания мочи, анатомо-топографическими особенностями нижних мочевыводящих путей или возрастной недостаточностью функции яичников. Вместе с тем определенную роль в патогенезе недержания мочи играет изменение функционального состояния почек. В ряде случаев нарушение контроля над мочеиспусканием сочетается с избыточным образованием мочи или инверсией суточного ритма мочеобразования за счет снижения реабсорбции ионов натрия в толстом восходящем отделе петли Генле. У части пациенток удалось нормализовать транспорт ионов в почке, диурез, суточный ритм мочеобразования приемом десмопрессина и диклофенака натрия.

Цель - оценка клинического значение нормализации ритма мочеотделения в восстановлении контроля над мочеиспусканием у пациенток с различными типами недержания мочи.

Материалы и методы исследования. В исследование были включены 130 женщин с недержанием мочи, полиурией (объем мочи, выделенной за сутки, превышает 40 мл/кг массы тела) или никтурией (ночной диурез/ диурез за сутки более 0,33 при продолжительности ночного сна не менее 8 часов), 30 женщин с недержанием мочи без нарушений мочеобразования составили группу сравнения и 14 здоровых женщин - группу контроля. Средний возраст женщин основной группы составил 43,6 4 4,5 года, группы сравнения - 41,8 \pm 3,7 года, группы контроля $-39,4 \pm 6,3$ года $(p>0,05)$. Обследование женщин с недержанием мочи и контрольной группы проводилось при обычном пищевом и водном режимах. Дневник мочеиспускания вели не менее 7 дней. В дневнике мочеиспускания указывали время позыва к мочеиспусканию, объем выделенной мочи за одно мочеиспускание, время сна, время и количество выпитой жидкости. Пациенткам основной группы с целью оценки количества потерянной мочи проводили трехкратный тест с прокладкой в течение суток.

Результаты исследования. Применение диклофенака натрия и десмопрессина у пациенток с различными типами недержанием мочи, полиурией или никтурией способствует восстановлению контроля над мочеиспусканием, снижению количества потерянной мочи за счет нормализации диуреза и увеличения цистометрической емкости мочевого пузыря.
\end{abstract}

- Ключевые слова: недержание мочи; полиурия; никтурия; диклофенак натрия, десмопрессин.

\title{
CLINICAL EFFICIENCY OF DESMOPRESSIN AND CYCLOOXYGENASE INHIBITOR USE IN INCONTINENT WOMEN
}

\section{(c) N.A. Osipova ${ }^{1}$, D.A. Niauri ${ }^{1}$, A.M. Gzgzyan ${ }^{1,2}$}

${ }^{1}$ Saint Petersburg State University, Medical Faculty, St. Petersburg, Russia

${ }^{2}$ The Research Institute of Obstetrics, Gynecology and Reproductology named after D.O. Ott, St. Petersburg, Russia

For citation: Osipova NA, Niauri DA, Gzgzyan AM. Clinical efficiency of desmopressin and cyclooxygenase inhibitor use in incontinent women. Journal of Obstetrics and Women's Diseases. 2018;67(2):40-51. doi: 10.17816/JOWD67240-51

Received: 01.03.2018

Accepted: 03.04.2018

- Hypothesis/aims of study. Questions regarding the pathogenesis of urine incontinence and methods of treatment are actively discussed by gynecologists, urologists, and neuropathologists. Urine incontinence often has multifactor origins: the causes of urine incontinence are connected, as a rule, with the violation of urine continence functional mechanisms, 
anatomotopographical features of the lower urinary tract, or an premature ovarian failure. Simultaneously, changes in kidney function play a role in the pathogenesis of urine incontinence. In some cases, urine incontinence is combined with urine overproduction or inversion of the circadian rhythm of renal function owing to a decrease in the reabsorption of sodium ions in the thick ascending limb of the loop of Henle. In some patients, we successfully normalized ion transport, diuresis, and circadian rhythm of urine production by desmopressin or diclofenac administration. The present analysis was undertaken to evaluate the clinical efficiency of desmopressin and diclofenac in incontinent patients with nocturnal polyuria and polyuria.

Study design, materials, and methods. In total, 130 patients with complaints of urinary incontinence, polyuria (24-h urine volume of $40 \mathrm{~mL} / \mathrm{kg}$ bodyweight or above), or nocturnal polyuria (nocturnal volume $/ 24-\mathrm{h}$ urine volume of 0.33 or above); 30 incontinent women without polyuria or nocturnal polyuria (comparison group); and 14 control subjects were included. The mean patient age was $43.6 \pm 4.5$ years $(41.8 \pm 3.7$ years in the comparison group and $39.4 \pm 6.3$ years in the control group, $p>0.05$ ). All the participants performed seven days of urine collection to determine the voided volumes. Patients with polyuria or nocturnal polyuria performed the 3-fold 24-h pad-test. Patients with polyuria and nocturnal polyuria were examined twice: in the initial state and one month after the start of treatment with the optimal dose of diclofenac or desmopressin (Minirin).

Results. The use of both diclofenac or desmopressin in patients with various types of urine incontinence, polyuria, or nocturia decreased the volume of voided urine because of the normalization of diuresis and an increase in cystometric bladder capacity.

- Keywords: urine incontinence; polyuria; nocturia; sodium diclofenac; desmopressin.

\section{Введение}

Распространенность недержания мочи в популяции остается значительной, с этой проблемой сталкивается около $10 \%$ женщин молодого возраста, 30-60 \% среднего возраста и 70-80\% резидентов домов престарелых [1-7]. В связи с этим продолжается поиск новых патогенетически обоснованных, эффективных методов лечения расстройств мочеиспускания у женщин.

Вопросы патогенеза и способы лечения недержания мочи у женщин активно обсуждаются гинекологами, урологами и невропатологами. Недержание мочи чаще всего имеет мультифакторное происхождение $[1,4,5,8]:$ причины возникновения инконтиненции связывают, как правило, с нарушением функциональных механизмов удержания мочи, анатомо-топографическими особенностями нижних мочевыводящих путей или возрастной недостаточностью функции яичников. Вместе с тем определенную роль в патогенезе недержания мочи играет изменение функционального состояния почек. В ряде случаев нарушение контроля над мочеиспусканием сочетается с избыточным образованием мочи или инверсией суточного ритма мочеобразования за счет снижения реабсорбции ионов натрия в толстом восходящем отделе петли Генле $[9,10]$. Реабсорбция $\mathrm{Na}^{+}, \mathrm{K}^{+}, \mathrm{Ca}^{2+}, \mathrm{Mg}^{2+}, \mathrm{Cl}^{-}$в этом отделе нефрона увеличивается при активации $\mathrm{V}_{2}$-рецепторов вазопрессином [11]. У части пациенток удается нормализовать транспорт ионов в почке, диурез, суточный ритм мочеобразования приемом синтетического аналога антидиуретического гормона - десмопрессина [12], однако в ряде случаев лучший эффект достигается при применении ингибитора синтеза простагландинов - диклофенака натрия [13].

Задачей настоящего исследования явилась оценка клинического значения нормализации ритма мочеотделения в восстановлении контроля над мочеиспусканием у пациенток с различными типами недержания мочи.

\section{Материалы и методы исследования}

В исследование были включены 130 женщин с недержанием мочи, полиурией (объем мочи, выделенной за сутки, превышает 40 мл/кг массы тела) или никтурией (ночной диурез/диурез за сутки более 0,33 при продолжительности ночного сна не менее 8 часов) [14], 30 женщин с недержанием мочи без нарушений мочеобразования составили группу сравнения и 14 здоровых женщин - группу контроля. Поскольку возраст и ассоциированные с ним изменения функции почек (скорость клубочковой фильтрации, концентрационная способность, чувствительность к гормональной регуляции) оказывают влияние на процессы образования мочи и после 50 лет количество мочи, выделенной ночью, удваивается, а к 60 годам и старше отмечается выраженная депрессия показателей, отражающих функционирование почек, в исследование были включены женщины моложе 50 лет $[15,16]$. Средний возраст женщин основной группы составил 43,6 \pm 4,5 года, группы сравнения - 41,8 $\pm 3,7$ года, группы контроля $-39,4 \pm 6,3$ года $(p>0,05)$. Для унификации результатов исследования мы считали 
необходимым применить следующие критерии включения и исключения.

Критерии включения в основную группу:

1) жалобы на недержание мочи вследствие физической нагрузки и/или позыва к мочеиспусканию в сочетании с полиурией и никтурией;

2) способность заполнять опросники, вести дневники мочеиспускания, готовность приезжать на осмотры в назначенное время.

Критерии включения в группу сравнения:

1) жалобы на недержание мочи вследствие физической нагрузки и/или позыва к мочеиспусканию без полиурии и никтурии;

2) способность заполнять опросники, вести дневники мочеиспускания, готовность приезжать на осмотры в назначенное время.

Критерии включения в контрольную группу:

1) отсутствие жалоб на недержание мочи вследствие физической нагрузки и/или позыва к мочеиспусканию, никтурии, полиурии;

2) способность заполнять опросники, вести дневники мочеиспускания, готовность приезжать на осмотры в назначенное время.

Критерии исключения для всех групп:

1) гистерэктомия и операции реконструкции тазового дна в анамнезе;

2) ранее перенесенное хирургическое лечение по поводу недержания мочи или опущения внутренних половых органов, пролапс тазовых органов II-IV стадий по системе POP-Q (ICS, 1996) в настоящее время;

3) психические заболевания, в том числе первичная психогенная полидипсия;

4) неврологические заболевания, сопровождающиеся нейрогенной дисфункцией мочевого пузыря;

5) заболевания сердечно-сосудистой системы, включая артериальную гипертензию;

6) заболевания эндокринной системы, глюкозурия;

7) заболевания почек и инфекционно-воспалительные заболевания мочевыводящих путей в стадии обострения, бактериурия;

8) урологические заболевания, при которых вероятно нарушение акта мочеиспускания;

9) прием любых лекарственных средств и гормональных препаратов в течение последних трех месяцев до начала обследования;

10) возраст старше 50 лет.

На основании данных комплексного стандартизованного алгоритма обследования, включа- ющего клиническое обследование, эхографию уретровезикального сегмента и уродинамическое исследование, у 45 женщин было диагностировано стрессовое недержание мочи, у 33 императивное и у $52-$ смешанное.

Обследование женщин с недержанием мочи и контрольной группы проводилось при обычном пищевом и водном режимах. Дневник мочеиспускания вели не менее 7 дней. В дневнике мочеиспускания указывали время позыва к мочеиспусканию, объем выделенной мочи за одно мочеиспускание, время сна, время и количество выпитой жидкости. Пациенткам основной группы с целью оценки количества потерянной мочи выполняли трехкратный тест с прокладкой в течение суток. Количество потерянной мочи рассчитывали по формуле $m_{2}-m_{1} \cdot n$, где $m_{2}-$ масса использованных за сутки прокладок; $m_{1}-$ масса неиспользованной прокладки; $n-$ количество использованных прокладок.

Пациентки с полиурией и никтурией обследовались повторно через 4 недели после приема терапевтической дозы ингибитора синтеза простагландинов диклофенака натрия (Диклофенак) или синтетического аналога антидиуретического гормона - десмопрессина (Минирина). Доза препаратов подбиралась индивидуально. Начальная суточная доза Диклофенака 25 мг 2 раза в день через 7 дней при отсутствии эффекта увеличивалась до 50 мг 2 раза в день и далее через 7 дней до 75 мг 2 раза в день; начальная доза Минирина 100 мкг 1 раз в день через 7 дней при отсутствии эффекта увеличивалась до 200 мкг и далее через 7 дней до 400 мкг 1 раз в день. На основании критериев диагностики полиурии и никтурии [11] эффект от терапии считали полным, если скорость мочеотделения становилась менее 40 мл/кг/24 часа при полиурии или индекс ночной полиурии становился менее 33 \% при никтурии; эффект от терапии считали частичным, если при полиурии скорость мочеотделения достоверно снижалась относительно исходных показателей, но была выше 40 мл/кг/24 часа, а при никтурии индекс ночной полиурии достоверно снижался относительно исходных показателей, но был выше 33 \%.

Для оценки межгрупповых различий при статистической обработке полученных результатов применяли $t$-критерий Стьюдента. Данные различия считали достоверными при вероятности ошибки $p<0,05$. Приведенные в работе таблицы содержат значения $M \pm m$. 


\section{Результаты}

На основании анализа дневников мочеиспускания было установлено, что у 59 женщин с жалобами на недержание мочи увеличена суточная продукция мочи, а у 71 имеет место увеличение ночного диуреза (никтурии). Полиурия выявлена у $37,8 \pm 7,2 \%$ женщин со стрессовым недержанием мочи, у 45,5 $\pm 8,7 \%$ с императивным и у $50,0 \pm 6,9 \%$ со смешанным $(p>0,05)$, никтурия - у 62,2 $\pm 7,2$ \% женщин со стрессовым недержанием мочи, у 51,5 \pm 8,7 \% с императивным и у 50,0 $\pm 6,9 \%$ со смешанным ( $p>0,05)$.

$\mathrm{y}$ пациенток с полиурией и никтурией независимо от типа недержания мочи суточный и ночной диурез, количество мочеиспусканий за сутки и в ночное время значительно превышали аналогичные показатели в контрольной группе и в группе сравнения. Количество эпизодов недержания мочи у пациенток с полиурией и никтурией было достоверно выше при императивном и смешанном типе недержания (табл. 1-4).

Применение Минирина и Диклофенака привело к уменьшению диуреза, количества мочеиспусканий во всех группах независимо от типа инконтиненции, исследуемые показатели стали сопоставимы с аналогичными показателями в группе сравнения. Количество эпизодов недержания мочи и количество потерянной мочи достоверно снизилось у пациенток с императивным и смешанным типом недержания мочи. У пациенток со стрессовым недержанием мочи, хотя количество эпизодов недержания мочи не изменилось, количество потерянной мочи достоверно снизилось (см. табл. 1-4).

В ходе цистометрии через 4 недели использования Диклофенака у пациенток с императивным недержанием мочи объем наполнения мочевого пузыря при первом позыве увеличился с $52 \pm 14$ до $94 \pm 12$ мл ( $p>0,05)$; у пациенток со смешанным недержанием мочи этот же показатель увеличился с $63 \pm 13$ до $104 \pm 11$ мл $(p>0,05)$; в ходе цистометрии через 4 недели использования Минирина у пациенток с императивным недержанием мочи объем наполнения мочевого пузыря при первом позыве увеличился с $54 \pm 11$ до $98 \pm 17$ мл ( $p>0,05)$; у пациенток со смешанным недержанием мочи этот же показатель увеличился с $58 \pm 15$ до $112 \pm 18$ мл $(p>0,05)$.

\section{Обсуждение результатов}

Недержанием мочи страдает около $10 \%$ женщин молодого возраста, 30-60 \% среднего возраста и 70-80 \% резидентов домов престарелых
$[1,2,5-7,17]$. Повышенный интерес к этой проблеме с конца 90-х гг. ХХ в. был обусловлен тем, что появившиеся методы исследования, в частности уродинамические, показали клиническую неоднородность пациентов с точки зрения этиологии и патогенеза симптома. Это привело к необходимости пересмотра и стандартизации терминов. В 2002 г. комитетом Международного общества по проблеме удержания мочи была предложена классификация недержания мочи: стрессовое, императивное и смешанное [18].

В основе консервативной терапии недержания мочи лежит воздействие на холино- и адренорецепторы нижних мочевыводящих путей, дополнительно применяются трициклические антидепрессанты, обладающие центральным и периферическим антихолинергическим действием, антагонисты кальция, активаторы калиевых каналов, препараты половых стероидов [19-22]. Вместе с тем появились данные об эффективном применении в ряде случаев антидиуретического гормона [23-28] как для лечения недержания мочи, так и для коррекции избыточного мочеобразования у пациентов с никтурией в основном пожилого возраста, поскольку при старении снижается уровень секреции антидиуретического гормона, что ведет, наряду со снижением концентрационной способности почек, к избыточному мочеобразованию [16]. В 2011 г. Европейская ассоциация урологов одобрила применение синтетического аналога антидиуретического гормона десмопрессина для лечения никтурии [29]. Еще ранее этот препарат стали применять для лечения энуреза у детей [30, 31].

При обследовании 277 женщин с недержанием мочи нами было установлено, что избыточное мочеобразование характерно не только для пожилых женщин, но и для женщин репродуктивного и пременопаузального возраста и проявляется в двух клинических вариантах - полиурией (у 25,0 4 4,4 \% пациентов) и никтурией (у 62,5 $\pm 4,9 \%$ пациентов) [32]. Далее нами было показано, что увеличение диуреза в этой группе пациенток, независимо от типа недержания мочи, обусловлено снижением реабсорбции ионов натрия и осмотически связанной с ним воды в толстом восходящем отделе петли Генле, а не с уменьшением проницаемости стенки собирательных трубок для воды $[9,10]$.

Действие антидиуретического гормона локализовано не только в собирательных трубках, 


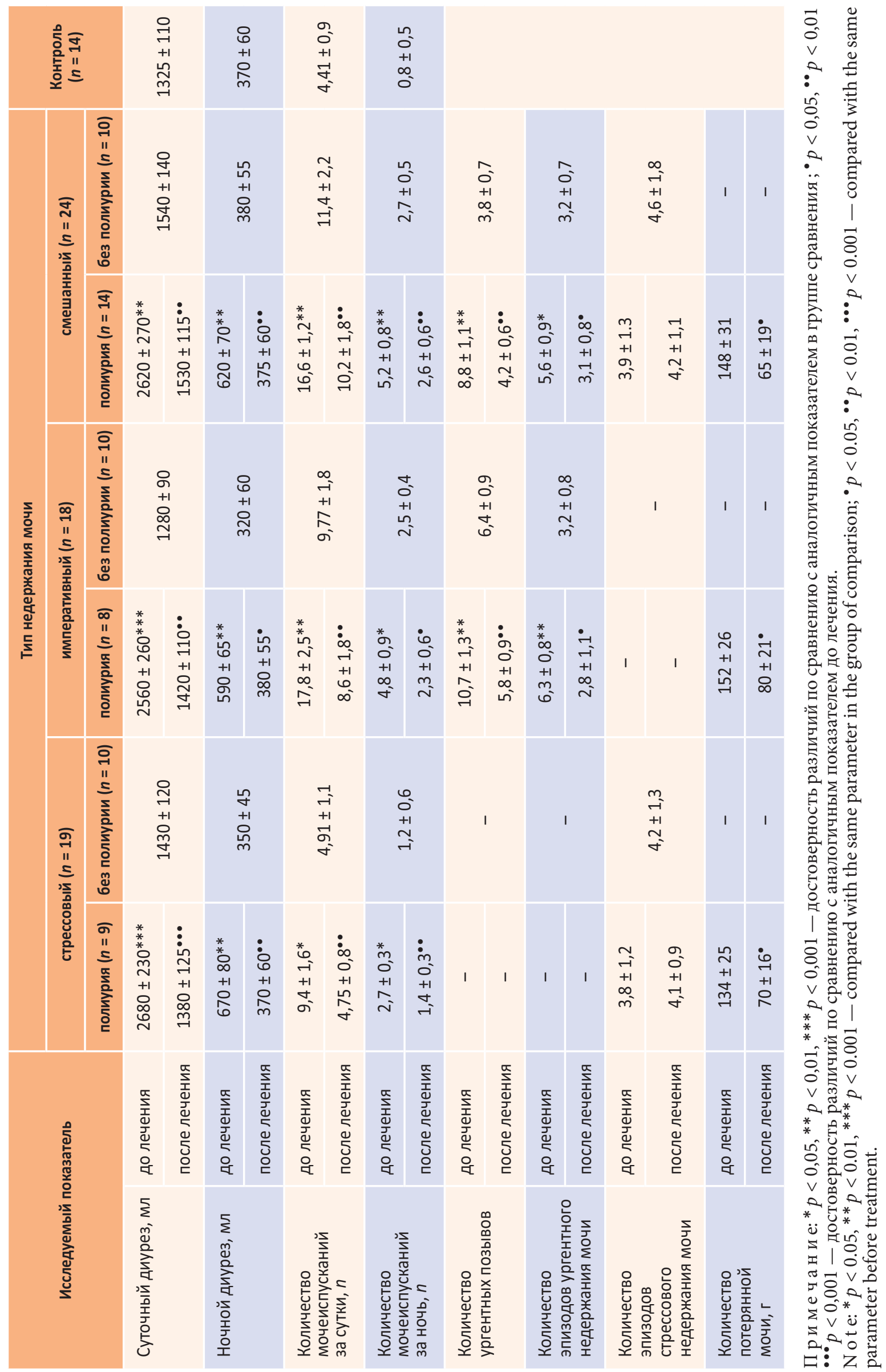




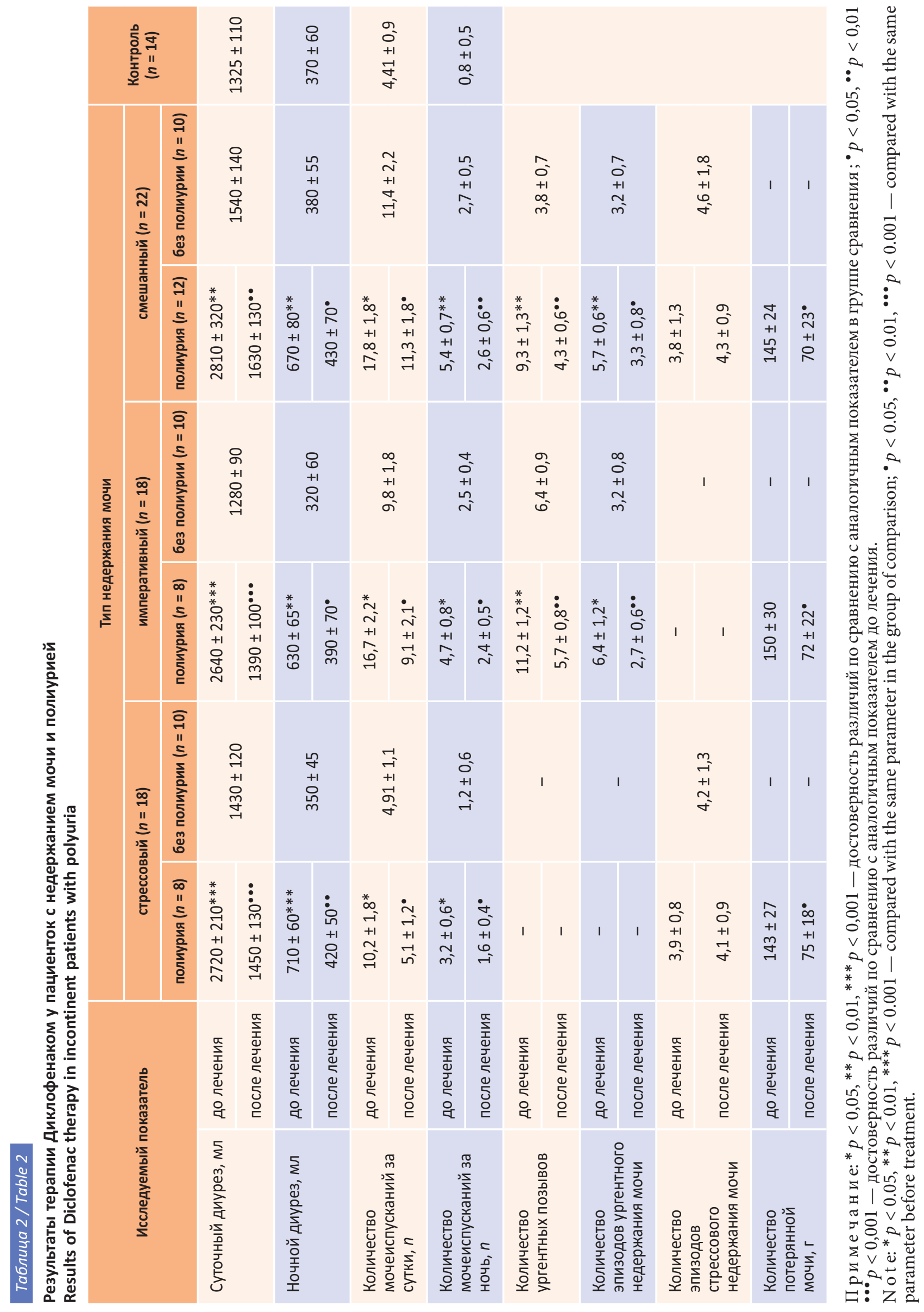




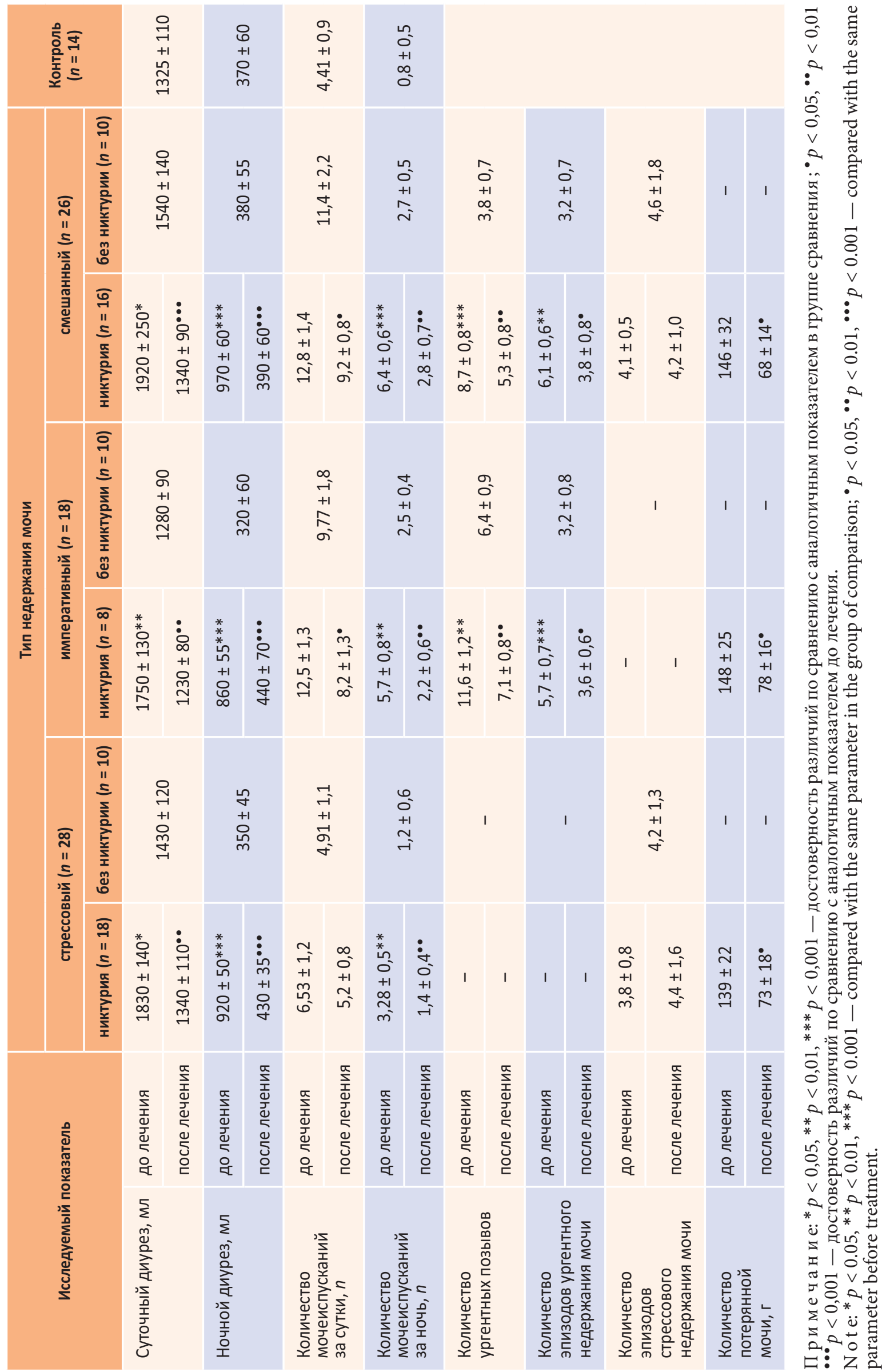




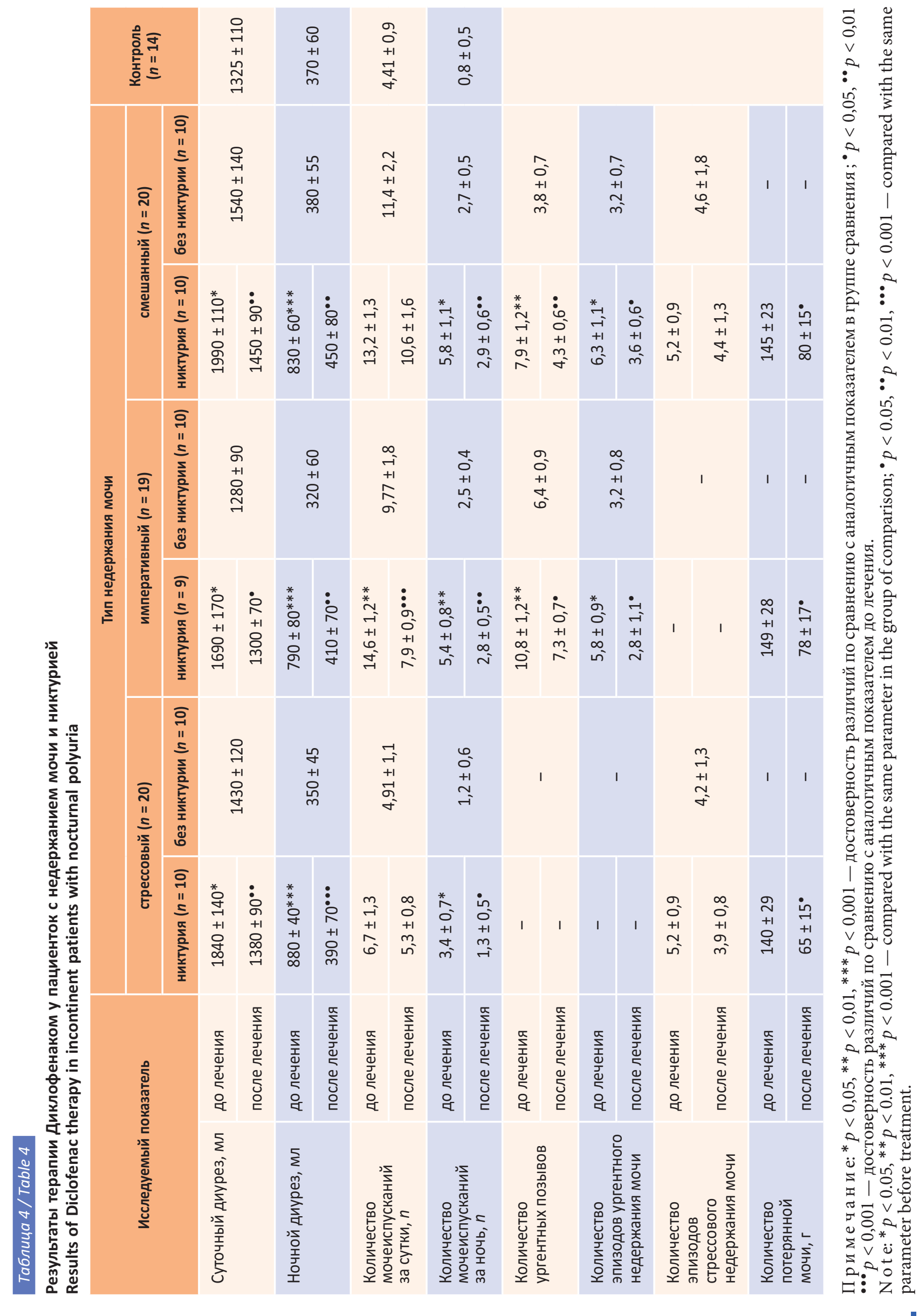


где связывание с $\mathrm{V}_{2}$-рецепторами приводит к реабсорбции осмотически свободной воды $[33,34]$. Активация $\mathrm{V}_{2}$-рецепторов вазопрессином в клетках толстого восходящего отдела петли Генле вызывает увеличение внутриклеточной концентрации цАМФ, и вследствие этого возрастает реабсорбция ионов натрия, калия, кальция, магния, хлора [11]. Нами было показано, что применение десмопрессина у пациенток с недержанием мочи, полиурией или никтурией оказывает нормализующее действие на транспорт ионов и позволяет добиться положительного эффекта в виде восстановления физиологического ритма мочеобразования у 73,8 \pm 4,8 \% пациенток [12]. Однако в ряде случаев применение десмопрессина было не эффективно. Отсутствие эффекта от терапии десмопрессином могло быть связано с локальным выделением веществ, противостоящих действию этого гормона, в частности простагландина $\mathrm{E}_{2}$. Простагландины наиболее интенсивно секретируются в структурах мозгового вещества почки [35]. В клетках толстого восходящего отдела петли Генле они снижают реабсорбцию ионов $\mathrm{Na}, \mathrm{Cl}$ [36] и ионов магния [37]. Кроме того, у пациенток с недержанием мочи выявлена повышенная экскреция простагландина $\mathrm{E}_{2}$ с мочой [38-40]. В группе пациенток с недержанием мочи, полиурией или никтурией и отсутствием эффекта от применения десмопрессина был использован блокатор синтеза простагландинов диклофенак натрия, что позволило добиться положительного эффекта и нормализовать ритм мочеобразования у 75,0 \pm 6,5 \% пациенток [13].

Приведенные выше данные подтверждают предположение о полиморфности нарушений механизмов мочеобразования при недержании мочи у женщин: реабсорбция ионов в толстом восходящем отделе петли Генле могла быть нарушена из-за повышенного образования простагландинов в этом отделе нефрона, поэтому была эффективна терапия диклофенаком натрия; у других пациенток, по всей видимости, уровень образования простагландинов неизменен, но может быть нарушена рецепция гормона вазопрессина клетками толстого восходящего отдела петли Генле, в таком случае можно предположить, что более эффективен будет синтетический аналог вазопрессина десмопрессин. Вместе с тем представляет интерес оценка клинического значения нормализации ритма мочеотделения в восстанов- лении контроля над мочеиспусканием у пациенток с различными типами недержания мочи.

Полученные нами данные свидетельствуют о том, что снижение суточного и ночного диуреза при применении Минирина и Диклофенака хотя и не приводит к уменьшению количества эпизодов стрессового недержания мочи, однако способствует снижению количества потерянной мочи. У пациенток с императивным и смешанным типом недержания положительный клинический эффект достигается как за счет снижения мочеобразования, так и за счет снижения количества эпизодов ургентного недержания мочи вследствие увеличения цистометрической емкости мочевого пузыря.

При применении Диклофенака этот эффект может достигаться как за счет снижения синтеза простагландинов в уротелии [45], так и опосредованно за счет блокады C-афферентных волокон, имеющих рецепторы к простагландинам [46]. При применении Минирина увеличение цистометрической емкости достигается за счет воздействия на нейроны центра мочеиспускания: нейроны $\mathrm{E}_{2}$-типа центра мочеиспускания непосредственно регулируют активность мочевого пузыря, нейроны $\mathrm{E}_{1}$-типа и I типа модулируют активность нейронов $\mathrm{E}_{2}$-типа, оказывая стимулирующее или блокирующее действие. Минирин снижает сократительную активность мочевого пузыря, ингибируя нейроны $\mathrm{E}_{1}$-типа или активируя нейроны I типа [47].

\section{Заключение}

Таким образом, применение Диклофенака и Минирина у пациенток с различными типами недержания мочи, полиурией или никтурией способствует восстановлению контроля над мочеиспусканием, снижению количества потерянной мочи за счет нормализации диуреза и увеличения цистометрической емкости мочевого пузыря у пациенток с императивным и смешанным типами недержания мочи.

\section{Дополнительная информация}

Вклад в исследование:

Н.А. Осипова - концепция исследования, сбор и обработка материалов, анализ полученных данных, написание текста.

Д.А. Ниаури - концепция исследования, анализ полученных данных.

A.M. Гзгзян - концепция исследования, анализ полученных данных. 


\section{Литература}

1. Аполихина И.А., Ромих В.В., Андикян В.М. Современные принципы консервативного лечения недержания мочи у женщин // Урология. - 2005. - № 5. - С. 72-75. [Ароlikhina IA, Romikh VV, Andikyan VM. Current principles of conservative treatment of urine incontinence in women. Urologiia. 2005;(5):72-75. (In Russ.)]

2. Балан В.Е., Анкирская А.С., Есесидзе 3.Т., Муравьева В.В. Патогенез атрофического цистоуретрита и различные виды недержания мочи у женщин в климактерии // Consilium medicum. - 2001. - T. 3. - № 7. - C. 326-331. [Balan VE, Ankirskaya AS, Esesidze ZT, Murav'eva VV. Pathogenesis of atrophic cystourethritis and various types of urinary incontinence in women in menopause. Consilium medicum. 2001;3(7):326-331. (In Russ.)]

3. Неймарк А.И., Раздорская М.В. Актуальные проблемы урогинекологии. Недержание мочи у женщин (лекция) // Акушерство, гинекология и репродукция. 2011. - T. 5. - № 4. - C. 27-36. [Neimark Al, Razdorskaya MV. Topical problems in urogynaecology. Urinary incontinence in women (lecture). Akusherstvo, ginekologiya i reproduktsiya. 2011;5(4):27-36. (In Russ.)]

4. Пушкарь Д.Ю., Щавелева О.Б. Диагностика и результаты лечения смешанных форм недержания мочи у женщин // Урология. - 2005. - № 3. - C. 22-25. [Pushkar DY, Schaveleva OB. Mixed forms of urinary incontinence in women: diagnosis and treatment. Urologiia. 2005;(3):22-25. (In Russ.)]

5. Hampel C, Wienhold D, Benken N, et al. Prevalence and natural history of female incontinence. Eur Urol. 1997;32(Suppl 2):3-12.

6. Rose A, Thimme A, Halfar C, et al. Severity of urinary incontinence of nursing home residents correlates with malnutrition, dementia and loss of mobility. Urol Int. 2013;91(2):165-169. doi: 10.1159/000348344.

7. Turan C, Zorlu CG, Ekin $M$, et al. Urinary incontinence in women of reproductive age. Gynecol Obstet Invest. 1996;41(2):132-134. doi: 10.1159/000292059.

8. Jonas U. Introduction and conclusions. Eur Urol. 2005;4(suppl 1):1-4.

9. Осипова Н.А., Гулиев Б.Г., Ниаури Д.А., Гзгзян А.М. Осморегулирующая функция почек при различных типах недержания мочи у женщин // Экспериментальная и клиническая урология. - 2016. № 3. - C. 144-149. [Osipova NA, Guliev BG, Niauri DA, Gzgzyan AM. The osmoregulatory function of kidneys in women with different types of urinary incontinence. Experimental \& Clinical Urology. 2016;(3):144-149. (In Russ.)]

10. Осипова Н.А., Ниуари Д.А., Гзгзян А.М. Ионорегулирующая функция почек при различных типах недержания мочи у женщин // Журнал акушерства и женских болезней. - 2016. - № 2. - C. 44-53. [Osipova NA, Niauri DA, Gzgzyan AM. Renal ionoregulation function in incontinent women. Journal of Obstetrics and Women's Diseases. 2016;(2):44-53. (In Russ.)]. doi: 10.17816/JOWD65244-53.

11. De Rouffignac C. Multihormonal regulation of nephron epithelia: achieved through combinational mode? Am J Physiol. 1995;269(4 Pt 2):R739-748. doi: 10.1152/ ajpregu.1995.269.4.R739.

12. Осипова Н.А., Ниаури Д.А., Гзгзян А.М. Патогенетическое обоснование возможности применения антидиуретического гормона при недержании мочи у женщин // Журнал акушерства и женских болезней. - 2017. - Т. 66. № 4. - C. 68-78. [Osipova NA, Niauri DA, Gzgzyan AM. Pathogenetic justification of possibility of antidiuretic hormone use in incontinent women. Journal of Obstetrics and Women's Diseases. 2017;66(4):68-78. (In Russ.)]. doi: 10.17816/JOWD66468-78.

13. Осипова Н.А., Ниаури Д.А., Гзгзян А.М. Опыт применения ингибитора циклооксигеназы для коррекции избыточного мочеобразования при недержании мочи у женщин // Журнал акушерства и женских болезней. - 2018. - Т. 67. - № 1. - C. 47-57. [Osipova NA, Niauri DA, Gzgzyan AM. Experience of cyclooxygenase inhibitor use for correction of urine overproduction in incontinent women. Journal of Obstetrics and Women's Diseases. 2018;67(1):47-57. (In Russ.)]. doi: 10.17816/JOWD67147-57.

14. van Kerrebroeck $P$, Abrams $P$, Chaikin D, et al. The standardisation of terminology in nocturia: report from the Standardisation Sub-committee of the International Continence Society. Neurourol Urodyn. 2002;21(2):179-183. doi: 10.1002/nau.10053.

15. Борисов И.А., Перов Ю.Л. Старческая почка // Нефрология / Под ред. И.Е. Тареевой. - М.: Медицина, 2001. C. 509-525. [Borisov IA, Perov YL. Old kidney. In: Nephrology. Ed by I.E. Tareeva. Moscow: Meditsina; 2001. P. 509-525. (In Russ.)]

16. Тюзиков И.А., Греков Е.А., Апетов С.С., и др. Ноктурия: современные гендерные аспекты эпидемиологии, патогенеза и диагностики // Экспериментальная и клиническая урология. - 2013. - № 3. - С. 113-122. [Tyuzikov IA, Grekov EA, Apetov SS, et al. Nocturia: modern gender aspects of epydemiology, pathogenesis and diagnostics. Experimental \& clinical urology. 2013;(3):113-122. (In Russ.)]

17. Jerez-Roig J, Santos MM, Souza DL, et al. Prevalence of urinary incontinence and associated factors in nursing home residents. Neurourol Urodyn. 2016;35(1):102-107. doi: 10.1002/nau.22675.

18. Abrams $P$, Cardozo L, Fall $M$, et al. The standardisation of terminology of lower urinary tract function: Report from the standardisation sub-committee of the international continence society. Am J Obstet Gynecol. 2002;187(1):116-126. doi: 10.1067/mob.2002.125704.

19. Мазо Е.Б. Медикаментозное лечение гиперактивного мочевого пузыря // РМЖ. - 2004. - Т. 12. - № 8. C. 522-526. [Mazo EB. Drug treatment of a hyperactive 
bladder. Russkii meditsinskii zhurnal. 2004;12(8):522-526. (In Russ.)]

20. Bissada NK, Finkbeiner AE. Urologic manifestations of drug therapy. Urol Clin North Am. 1988;15(4):725-736.

21. Sellers DJ, Chapple CR, Chess-Williams R. Potential therapeutic targets for treatment of the overactive bladder. World J Urol. 2001;19(5):307-311. doi: 10.1007/pl00007102.

22. Wein AJ. Pharmacological agents for the treatment of urinary incontinence due to overactive bladder. Expert Opin Investig Drugs. 2005;10(1):65-83. doi: 10.1517/13543784.10.1.65.

23. Han YK, Lee WK, Lee SH, et al. Effect of Desmopressin with Anticholinergics in Female Patients with Overactive Bladder. Korean J Urol. 2011;52(6):396. doi: 10.4111/ kju.2011.52.6.396.

24. Hilton P, Stanton SL. The Use of Desmopressin (DDAVP) in Nocturna Urinary Frequency in the Female. $\mathrm{Br} J$ Urol. 1982;54(3):252-255. doi: 10.1111/j.1464-410X.1982. tb06969.x.

25. Jeong JY, Kim SJ, Cho HJ, et al. Influence of type of nocturia and lower urinary tract symptoms on therapeutic outcome in women treated with desmopressin. Korean J Urol. 2013;54(2):95-99. doi: 10.4111/kju.2013.54.2.95.

26. Lee HW, Choo MS, Lee JG, et al. Desmopressin is an effective treatment for mixed nocturia with nocturnal polyuria and decreased nocturnal bladder capacity. I Korean Med Sci. 2010;25(12):1792-1797. doi: 10.3346/ jkms.2010.25.12.1792.

27. Mun JH, Kim SO, Yu HS, et al. Effects of desmopressin for the treatment of nocturnal polyuria in elderly women: impact on related sleep quality. Can Urol Assoc J. 2015;9(11-12):E770-774. doi: 10.5489/cuaj.3097.

28. Robinson D, Cardozo L, Akeson M, et al. Antidiuresis: a new concept in managing female daytime urinary incontinence. BJU Int. 2004;93(7):996-1000. doi: 10.1111/j.1464410X.2004.04768.x.

29. Тюзиков И.А., Калиниченко С.Ю., Ворслов Л.О., Тишова Ю.А. Дефицит вазопрессина как причина ноктурии и гормонально-метаболических нарушений у мужчин. Роль десмопрессина в их коррекции // Эффективная фармакотерапия. - 2015. - № 27. - С. 26-37. [Tyuzikov IA, Kalinichenko SY, Vorslov LO, Tishova YA. Vasopressin Deficiency as a Cause of Nocturia and Hormonal-Metabolic Disorders in Men. A Role of Desmopressin in Their Correction. Effektivnaya farmakoterapiya. 2015;(27):26-37. (In Russ.)]

30. Nørgaard JP, Pedersen EB, Djurhuus JC. Diurnal anti-diuretic-hormone levels in enuretics. J Urol. 1985;134(5):10291031.

31. Rittig S, Knudsen UB, Norgaard JP, et al. Abnormal diurnal rhythm of plasma vasopressin and urinary output in patients with enuresis. Am J Physiol Renal Physiol. 1989;256(4):F664F671. doi: 10.1152/ajprenal.1989.256.4.F664.

32. Осипова Н.А., Ниаури Д.А., Зиятдинова Г.М. Суточный ритм мочеобразования и мочеотделения при недержании мочи у женщин // Гинекология. - 2015. - Т. 17. -
№ 2. - C. 74-76. [Osipova NA, Niaury DA, Ziyatdinova GM. The circadian rhythm of urine formation in incontinent women. Gynecology. 2015;17(2):74-76. (In Russ.)]

33. Иванова Л.Н. Вазопрессин: клеточные и молекулярные аспекты его антидиуретического действия // Вестник Российской академии медицинских наук. - 1999. № 3. - C. 40-45. [Ivanova LN. Vasopressin: cellular and molecular aspects of its antidiuretic effect. Annals of the Russian Academy of Medical Sciences. 1999;(3):40-45. (In Russ.)]

34. Sabolić I, Brown D. Water transport in renal tubules is mediated by aquaporins. Clin Investig. 1994;72(9):698-700. doi: 10.1007/bf00212993.

35. Наточин Ю.В. Физиология водно-солевого обмена и почки. - СПб., 1993. [Natochin YV. Physiology of water-salt metabolism and kidneys. Saint Petersburg; 1993. (In Russ.)]

36. Aarab L, Siaume-Perez S, Chabardes D. Cell-specific coupling of PGE2 to different transduction pathways in arginine vasopressin- and glucagon-sensitive segments of the rat renal tubule. Br J Pharmacol. 1999;126(4):1041-1049. doi: 10.1038/sj.bjp.0702390.

37. Гончаревская О.А., Наточин Ю.В. Простагландин $E_{2}$ угнетает реабсорбцию магния в начальном отделе дистального отдела нефрона тритона // Доклады PAH. - 1994. - T. 337. - № 1. - C. 125-127. [Goncharevskaja OA, Natochin YV. Prostaglandin E 2 inhibits the reabsorption of magnesium in the initial section of the distal nephron nephritis. Doklady RAN. 1994;337(1):125-127. (In Russ.)]

38. Cho KJ, Kim HS, Koh JS, Kim JC. Changes in urinary nerve growth factor and prostaglandin $E_{2}$ in women with overactive bladder after anticholinergics. Int Urogynecol $\mathrm{J}$. 2013;24(2):325-330. doi: 10.1007/s00192-012-1854-4.

39. Kim JC, Park EY, Seo SI, et al. Nerve Growth Factor and Prostaglandins in the Urine of Female Patients With Overactive Bladder. J Urol. 2006;175(5):1773-1776. doi: 10.1016/ s0022-5347(05)00992-4.

40. Marentette JO, Hurst RE, McHowat J. Impaired Expression of Prostaglandin $E_{2}$ (PGE2) Synthesis and Degradation Enzymes during Differentiation of Immortalized Urothelial Cells from Patients with Interstitial Cystitis/Painful Bladder Syndrome. PLoS One. 2015;10(6):e0129466. doi: 10.1371/ journal.pone.0129466.

41. Danon A, Zenser TV, Thomasson DL, Davis BB. Eicosanoid synthesis by cultured human urothelial cells: potential role in bladder cancer. Cancer Res. 1986;46(11):5676-5681.

42. Wong Y-HH, Zenser TV, Davis BB. Regulation of dog urothelial cell arachidonic acid release and prostaglandin $E_{2}$ synthesis. Carcinogenesis. 1989;10(9):1621-1627. doi: 10.1093/ carcin/10.9.1621.

43. Delaere KP, Debruyne FM, Moonen WA. The use of indomethacin in the treatment of idiopathic bladder instability. Urol Int. 1981;36(2):124-127. doi: 10.1159/000280402. 
44. Sprem M, Milicic D, Oreskovic S, et al. Intravesically administered ketoprofen in treatment of detrusor instability: cross-over study. Croat Med J. 2000;41(4):423-427.

45. Takagi-Matsumoto H, Ng B, Tsukimi Y, Tajimi M. Effects of NSAIDs on Bladder Function in Normal and Cystitis Rats: a Comparison Study of Aspirin, Indomethacin, and Ketoprofen. J Pharmacol Sci. 2004;95(4):458-465. doi: 10.1254/ jphs.FP0040098.
46. Tanaka I, Nagase K, Tanase K, et al. Improvement in neurogenic detrusor overactivity by peripheral $C$ fiber's suppression with cyclooxygenase inhibitors. J Urol. 2010;183(2):786-792. doi: 10.1016/j.juro.2009.09.071.

47. Iwasaki H, Koyama Y, Tanaka Y, et al. Modulation by desmopressin of neuronal activity in brainstem micturition center. Urology. 2004;63(5):994-998. doi: 10.1016/j. urology.2003.11.036.

- Адреса авторов для переписки (Information about the authors)

Наталья Анатольевна Осипова - ассистент кафедры акушерства, гинекологии и репродуктологии медицинского факультета ФГБОУ ВПО «Санкт-Петербургский государственный университет», Санкт-Петербург. E-mail: naosipova@mail.ru.

Дарико Александровна Ниаури - д-р мед. наук, заведующая кафедрой акушерства, гинекологии и репродуктологии медицинского факультета ФГБОУ ВПО «Санкт-Петербургский государственный университета».E-mail: d.niauri@mail.ru.

Александр Мкртичевич Гзгзян - д-р мед. наук, профессор кафедры акушерства, гинекологии и репродуктологии ФГБОУ ВПО «Санкт-Петербургский государственный университет», руководитель отделения вспомогательных репродуктивных технологий ФГБНУ «НИИ АГиР им. Д.О. Отта», Санкт-Петербург. E-mail: iagmail@ott.ru.
Natalya A. Osipova - Professor Assistant, PhD, St. Petersburg State University, Medical Faculty. Department of Obstetrics, Gynecology and Reproductology, St. Petersburg, Russia. E-mail: naosipova@mail.ru.

Dariko A. Niauri - MD, Prof. PhD, DMedSci, St. Petersburg State University, Medical Faculty, Department of Obstetrics, Gynecology and Reproductology, St. Petersburg, Russia. E-mail: d.niauri@mail.ru.

Alexander M. Gzgzyan - MD, PhD, DMedSci, St. Petersburg State University, Medical Faculty. Department of Obstetrics, Gynecology and Reproductology, Head of Reproductive Technologies Department. The Research Institute of Obstetrics, Gynecology and Reproductology named after D.O. Ott, St. Petersburg, Russia. E-mail: iagmail@ott.ru. 